\title{
About the Feasibility of Personalized Medicine in a Low Income Country?
}

\author{
Mona Mlika1,2, Emna Braham1,2, Soumaya Laabidi², Hamouda Boussen², Faouzi El Mezni1,2 \\ ${ }^{1}$ Department of Pathology, Abderrahman Mami Hospital, Ariana, Tunisia \\ ${ }^{2}$ Faculté de Médecine de Tunis, Université Tunis El Manar, Tunis, Tunisia \\ Email: mounamlika22@gmail.com
}

Received 26 March 2015; accepted 3 May 2015; published 8 May 2015

Copyright (C) 2015 by authors and Scientific Research Publishing Inc.

This work is licensed under the Creative Commons Attribution International License (CC BY). http://creativecommons.org/licenses/by/4.0/

(c) (i) Open Access

\section{Abstract}

Personalized medicine is the result of the research made on the field of molecular biology. Lung cancer was mainly concerned with the establishment of many oncogenic drivers. Nowadays, the pathologist is facing a dilemma because of the multiplicity of therapeutic targets and molecular ones and the small size of specimen performed. In fact, $75 \%$ of lung cancer are diagnosed on biopsic samples. According to the recommendations of the 2013 International Group Study of Lung Cancer (IASLC), sequential analyses of Epidermal growth factor (EGFR) and anaplastic lymphoma kinase (ALK) are necessary on adenocarcinomas, non small cell carcinomas not otherwise specified and non small cell carcinomas favor adenocarcinomas. Many interrogations are reported concerning the cost effectiveness of the diagnostic techniques and the targeted treatments. We wondered about the further feasibility of such a technique in a low income country by trying to explore the representativity of the samples. In our hospital, bronchoscopic biopsies are diagnostic in $75 \%$ of the cases. We receive a mean of 4 samples. $68 \%$ of the samples are tumoral. Immunohistochemistry is performed in $68 \%$ of the cases with a mean of 2 antibodies used and $8 \%$ of the biopsies are non interpretable because of the small size of specimen. Concerning transthoracic biopsies, $20 \%$ are non contributive because of necrosis or the small size. We receive a mean of 1 sample with a mean size of 6 millimeters. Immunohistochemistry is performed in $71 \%$ of the cases with the mean of 2 antibodies. In addition to the scientific, pharmacoeconomic and ethic problems induced by targeted therapies in low income countries, each institution should optimize the use of the specimen received and the technical conditions in order to be ready to answer to the IASLC recommendation.

\section{Keywords}

Personalized Medicine, Target Therapies, Low Income Country 


\section{Introduction}

Lung cancer is a major public health problem. It represents the first cancer death cause through the world and in Tunisia. Its incidence has increased from 17.6 cases per 100,000 persons in 1997 to 27.6 cases per 100,000 persons in 2003 according to the register of the Northern Tunisian region [1]. The median age of the patients is 60 years. The surgical resection is the mainstay treatment but is possible only in $20 \%$ to $30 \%$ of the cases and the prognosis of the patients remains poor with a 5-year survival of $40 \%$. The microscopic profile of lung cancer has changed this decade with the adenocarcinoma becoming the most frequent histologic subtype. Since 2000, we are facing a revolution in the microscopic classification, the identification of molecular and prognostic factors and the discovery of new therapeutic targets. The American Thoracic Society (ATS), the European Respiratory Society (ERS) and the International Group Study of Lung Cancer (IASLC) established a new classification for lung cancer in cytology, biopsies and surgical specimen [2]. This new classification integrated for the first time molecular recommendations dealing with the necessity of the research of Epidermal growth factor receptor (EGFR) mutations in adenocarcinoma, non small cell lung carcinoma favor adenocarcinoma and non small cell lung carcinoma not otherwise specified [2]. The better knowledge of lung cancer carcinogenesis led to a personalized medicine. Two facts induced this medicine: the contraindication of some treatments in some histologic subtypes like bevacizumab and pemetrexed in squamous cell carcinoma and the establishment of oncogenic driver-addict character of lung cancer [3]-[5]. The pathologist's role has changed since 2000 from a morphologic role to a theranostic role. In fact, since 1999, our knowledge concerning the molecular drivers of lung cancer has been improving. In 1999, only KRAS mutations were known and $75 \%$ of the molecular drivers were unknown. This percentage decreased in 2004 when the implication of the EGFR gene was established. From 2005 to 2012, many other oncogenic drivers has been discovered including ALK, PIK3CA, MET, BRAF, HER2, RET, ROS1, MEK [6]. We wondered about the further feasibility of such a technique in a low income country by trying to explore the representativity of the samples.

\section{The Major Oncogenic Drivers and Predictive Response Markers}

EGFR gene encodes for a transmembrane tyrosine kinase. Upon binding to its ligands, EGFR forms homodimers or heterodimers with other family members (ERBB2, ERBB3 or ERBB4), which inactivates intrinsic receptor tyrosine kinase activity and triggers auto-phosphorylation of specific tyrosine residues within their cytoplasmic regulatory domains [7]. These phosphorylated tyrosine residues activate several signaling pathways including mitogen-activated protein kinase (MAPK) pathway, phosphatidylinositol 3-kinase (PI3K/AKT pathway) and the signal transducer and activator of transcription pathways. The mutations of EGFR reach $40 \%$ in East Asians and 15\% in Caucasians [8]. The presence of an EGFR mutation predicts likelihood of response to TKI therapy, with an observed response rate of about $80 \%$ among individuals whose tumors harbor the mutation and only $10 \%$ among those whose tumors do not [9] [10].The most frequent mutations have been identified in exons 18, 19, 20 and 21. These mutations or deletions, mainly in exon 19, result in increased malignant cell survival, proliferation, growth, invasion, metastatic spread and tumor angiogenesis [11]. These activating mutations are more frequently observed in never smokers female with Asian ethnicity and an adenocarcinoma histologic subtype. The activation of EGFR induces a cascade phosphorylation of RAS (rat sarcoma viral oncogene), RAF (v-raf murine leukemia viral oncogenehomolog), MEK (murine thymoma viral oncogenehomolog), ERK (extra cellular-signal-regulated kinase), PI3K/AKT (phosphatidylinositol 3-kinase). These interactions induce proliferation, neo-angiogenesis and metastasis [12]. In the opposition to colon cancer where the negative predictive value of these mutations on the response to EGFR-TKI has been proved, the impact of these mutations in NSCLC is still debated with contradictory results [13]. In the opposition to the EGFR pathway, which is implicated in non smokers, KRAS pathway is activated in smokers with adenocarcinoma.

The anaplastic lymphoma kinase gene (ALK) is located on chromosome 2p23. This gene was originally established for its implication in the pathogenesis of inflammatory myofibroblastic tumor and ALK-positive anaplastic large-cell lymphomas [14]. The fusion of ALK with the echinoderm microtubule-associated protein-like 4 gene (EML4) was initially identified in 2007 [14]. This gene fusion is due to small inversions on chromosome arm 2p. Further publications reported other partners to ALK gene including TGF gene located at 3q12.2 and KIF5B located at 10p11.22 [15] [16]. At least eleven variants of the EML4-ALK fusion gene with the same breakpoint in ALK gene and different ones in EML4 gene have been reported.

ROS1 rearrangement is observed in $78 \%$ of non-smoker patients. It is only observed in adenocarcinomas. 
Other emerging biomarkers were discovered and include Her2 gene mutation observed in $4 \%$ of non small cell lung cancer and mainly in adenocarcinomas. BRAF mutations were also observed in $3 \%$ of the adenocarcinomas in smokers. Mutations of the PIK3CA gene were also discovered [17].

In addition to these therapeutic targets, response predictive markers have also been reported. Excision-repair cross-complementation group I (ERCC1) protein is implicated in the repair of DNA lesions secondary to the platinum-based treatment and is over-expressed in resistant tumors with controversial relevance [18]. Regulatory subunit 1 of ribonucleotide Reductase (RRM1) is implicated in the DNA synthesis and is targeted by the gemcitabin [19]. EGFR mutations have been reported to be associated to diverse response to treatment, such as the T790M mutation which is correlated to a drug resistance. KRAS mutations have been correlated to worse response to the tyrosine kinase inhibitors [20]. The over-expression of the P53 protein has also been associated to a chemoresistance [21]. Thymidilate synthase is an enzyme implicated in the DNA synthesis and is implicated in the resistance to the pemetrexed.

\section{Brainstorming: What Are the Targets and the Predictive Markers to Detect in Daily Practice?}

We are facing too many markers, therapeutic or predictive. Dealing with all these markers in daily practice is a brainstorming. Four major questions have to be answered: which is the tissue to use, what is the histologic subtype to select, what is the best strategy to use and what are the diagnostic techniques to perform?

Nowadays, it is consensual to search for molecular targets in adenocarcinoma, non small cell carcinoma (NOS) and non small cell carcinoma favor adenocarcinoma. Concerning the tissue to use, a pathology department deals with diverse cytologic (exfoliative or aspirative), biopsies and surgical specimen. Surgical specimen offers generally enough tumoral material to perform molecular tests but $70 \%$ of lung cancers are discovered on small biopsies. Molecular tests should interpreted in regard to the intratumoral hemerogeneity and the technical conditions particular to every laboratory. Concerning cytologic specimen and according to the IASLC recommendations, a ROSE evaluation (rapid on-set evaluation) should be performed by a cytopathologist in order to ensure the representativity of the samples. Then Diagnostic value of cytologic specimen is consensual. They are helpful in distinguishing small cell carcinoma from non small cell carcinoma in $100 \%$ of the cases and allows to distinguish adenocarcinoma from squamous cell carcinoma in $96 \%$ of the cases in comparison with the tissue analysis. The molecular testing on cytoblocs is becoming consensual but the utility of pposition remains debated with contraindicatory results. Concerning biopsies, they may be performed through bronchoscopy or through a trans-thoracic approach [22]. The diagnostic value of bronchoscopic biopsies has been proved in $75 \%$ to $95 \%$ of the tumors. A minimum of 3 samples are necessary. The limits of bronchoscopic biopsies are that $1 / 2$ to $2 / 3$ of the samples aren't tumoral and immunohistochemistry is performed in $40 \%$ of the cases dealing with the abuse of paraffin blocks. Transthoracic biopsies are useful in peripheral tumors. They necessitate 3 samples and their use is limited by the limited amount of tumoral cells. Using biopsies in molecular testing necessitates a quick formol fixation of 6 to 8 hours, the inclusion of the specimen in paraffin with a temperature of inclusion inferior to $60^{\circ} \mathrm{C}$. The pathologist has to perform morphologic analysis, immunohistochemical study and molecular study on the same small samples. This situation is challenging and necessitates a hierarchized strategy [22]. According to the College American Pathologists (CAP), the International Association for the Study of Lung Cancer (IASLC) and the Association for Molecular Pathology (MAP) (2013) recommendations, testing of EGFR mutations and ALK/EML4 translocation must be performed in stage IV NSCLC and is encouraged in stages I, II and III [23]. Paraffin included formol-fixed specimen and fresh specimen are used. Cytoblocks could also be used. Sequential testing is necessary with a maximum of a 15-day delay to furnish a result. Emerging biomarkers aren't explored and the testing for secondary mutations dealing with therapeutic resistance such as MET amplification (5\%), EGFR T790M mutation (49\%) and PIK3CA mutations (5\%) is not necessary [23].

Concerning the diagnostic techniques, we are facing a huge of techniques with variable sensitivities. According to the literature review, EGFR mutations are searched using a PCR amplification followed by sequencing and ALK-EML4 translocation is initially screened by immunohistochemistry followed by FISH analysis [24]. According to the 2011 INCA report concerning the non interpretable EGFR testing, the main cause is represented by the low level of tumoral cells followed by a non amplified DNA and the loss of blocs [25].

\section{What about the Further Molecular Utility of the Samples in Our Hospital?}

We wondered about the representativity of the samples in our hospital and we found that bronchoscopic biopsies 
are diagnostic in $75 \%$ of the cases. We receive a mean of 4 samples. $68 \%$ of the samples are tumoral. Immunohistochemistry is performed in $68 \%$ of the cases with a mean of 2 antibodies used and $8 \%$ of the biopsies are non interpretable because of the small size of specimen. Concerning transthoracic biopsies, $20 \%$ are non contributive because of necrosis or the small size. We receive a mean of 1 sample with a mean size of 6 millimeters. Immunohistochemistry is performed in $71 \%$ of the cases with the mean of 2 antibodies. In order to optimize further molecular tests and according to the IASLC recommendations, we should decrease the immunohistochemical studies in order to save the tumor material for further molecular testing. Besides, according to the IASLC recommendations, immunohistochemistry is necessary only in cases difficult to subtype and the diagnosis of primary adenocarcinoma is based on clinical and radiological features, the immunohistochemistry is performed only if there is confusing clinical features.

\section{Conclusions}

Today, we are facing 3 major challenges concerning personalized medicine: a scientific problem, a pharmacoeconomic problem and an ethical problem.

The first challenge is due to the lack of knowledge concerning the molecular profile of adenocarcinoma in our country.

The second problem is due to the cost of the diagnostic techniques and the cost of the treatment. In fact, diagnostic techniques are very expensive and a multidisciplinary effort must be done in order to choose the best technique with the best Cost/quality ratio. Besides, target treatments are very expensive and many authors wondered about their cost-effectiveness. A rapid look to the literature, allows us to know that these treatment must be used according to the molecular testing in order to be affordable [26]. In opposition to the cost of the techniques and the treatments, the financial budget of the Public Health ministry seems to be insufficient. In fact, the 2013 budget of the Public Minister Health is approximately 398,000 millions of dinars and the regular expenses reach 340,000 millions of dinars [27].

The third ethical problem is faced nowadays in European and American societies. In fact, it seems that whiteAmerican and rich people are more likely to be treated with these treatments [28].

\section{References}

[1] Public Health Minister of Tunisia (1999-2003) North Tunisia Cancer Register. 71.

[2] Travis, W.D., Brambilla, E., Noguchi, M., Nicholson, A.G., Geisinger, K., Yatabe, Y., et al. (2013) Diagnosis of Lung Cancer in Small Biopsies and Cytology: Implications of the 2011 International Association for the Study of Lung Cancer/American Thoracic Society/European Respiratory Society Classification. Archives of Pathology \& Laboratory Medicine, 137, 668-684. http://dx.doi.org/10.5858/arpa.2012-0263-RA

[3] Lee, A.L., Ng, V.W., Gao, S., Hedrick, J.L. and Yang, Y.Y. (2015) Injectable Biodegradable Hydrogels from Vitamin D-Functionalized Polycarbonates for the Delivery of Avastin with Enhanced Therapeutic Efficiency against Metastatic Colorectal Cancer. Biomacromolecules, 16, 465-475. http://dx.doi.org/10.1021/bm5015206

[4] Kim, H.S., Lee, G.W., Kim, J.H., Kim, H.Y., Kwon, J.H., Song, H.H., Kim, H.J., Jung, J.Y., Jang, G., Choi, D.R., Park, S.M., Shin, T.R., Lee, H.S. and Zang, D.Y. (2010) A Phase II Study of Pemetrexed and Carboplatin as a Salvage Therapy for Platinum-Pretreated Patients with Non-Small Cell Lung Cancer. Lung Cancer, 70, 71-76. http://dx.doi.org/10.1016/j.lungcan.2009.12.015

[5] Pao, W., Wang, T.Y., Riely, G.J., et al. (2005) KRAS Mutations and Primary Resistance of Lung Adenocarcinoma to Gefitinib or Erlotinib. PLoS Medicine, 2, e17. http://dx.doi.org/10.1371/journal.pmed.0020017

[6] Adamson, R.T. (2013) Biomarkers and Molecular Profiling in Non-Small Cell Lung Cancr: An Expanding Role and Its Managed Care Implications. American Journal of Managed Care, 19, S398-S407.

[7] Hynes, N.E. and Lane, H.A. (2005) ERBB Receptors and Cancer: The Complexity of Targeted Inhibitors. Nature Reviews Cancer, 5, 341-354. http://dx.doi.org/10.1038/nrc1609

[8] Suda, K., Tomizawa, K. and Mitsudomi, T. (2010) Biological and Clinical Significance of KRAS Mutations in Lung Cancer: An Oncogenic Driver That Contrasts with EGFR Mutation. Cancer and Metastasis Reviews, 29, 49-60. http://dx.doi.org/10.1007/s10555-010-9209-4

[9] Carr, L.L., Finigan, J.H. and Kern, J.A. (2011) Evaluation and Treatment of Patients with Non-Small Cell Lung Cancer. Medical Clinics of North America, 95, 1041-1054. http://dx.doi.org/10.1016/j.mcna.2011.08.001

[10] Mitsudomi, T. and Yatabe, Y. (2007) Mutations of the Epidermal Growth Factor Receptor Gene and Related Genes as Determinants of Epidermal Growth Factor Receptor Tyrosine Kinase Inhibitors Sensitivity in Lung Cancer. Cancer 
Science, 98, 1817-1824. http://dx.doi.org/10.1111/j.1349-7006.2007.00607.x

[11] Berardi, R., Santoni, M., Morgese, F., Ballatore, Z., Savini, A., Onofri, A., et al. (2013) Novel Small Molecule EGFR Inhibitors as Candidate Drugs in Non-Small Cell Lung Cancer. OncoTargets and Therapy, 6, 563-576. http://dx.doi.org/10.2147/OTT.S28155

[12] Cataldo, V.D., Gibbons, D.L., Pérez-Soler, R. and Quintás-Cardama, A. (2011) Treatment of Non-Small-Cell Lung Cancer with Erlotinib or Gefitinib. New England Journal of Medicine, 364, 947-955. http://dx.doi.org/10.1056/NEJMct0807960

[13] Mao, C., Qiu, L.X., Liao, R.Y., Du, F.B., Ding, H., Yang, W.C., et al. (2010) KRAS Mutations and Resistance to EGFR-TKIs Treatment in Patient with Non-Small Cell Lung Cancer: A Meta-Analysis of 22 Studies. Lung Cancer, 69, 272-278. http://dx.doi.org/10.1016/j.lungcan.2009.11.020

[14] Soda, M., Choi, Y.L., Enomoto, M., Takada, S., Yamashita, Y., Ishikawa, S., et al. (2007) Identification of the Transforming EML4-ALK Fusion Gene in Non-Small-Cell Lung Cancer. Nature, 448, 561-566. http://dx.doi.org/10.1038/nature05945

[15] Rikova, K., Guo, A., Zeng, Q., Possemato, A., Yu, J., Haack, H., et al. (2007) Global Survey of Phosphotyrosine Signaling Identifies Oncogenic Kinases in Lung Cancer. Cell, 131, 1190-1203. http://dx.doi.org/10.1016/j.cell.2007.11.025

[16] Takeuchi, K., Choi, Y.L., Togashi, Y., Soda, M., Hatano, S., Inamura, K., et al. (2009) KIF5B-ALK, a Novel Fusion Oncokinase Identified by Immunohistochemistry-Based Diagnostic System for ALK-Positive Lung Cancer. Clinical Cancer Research, 15, 3143-3149. http://dx.doi.org/10.1158/1078-0432.CCR-08-3248

[17] Pan, Y., Zhang, Y., Li, Y., Hu, H., Wang, L., Li, H., Wang, R., Ye, T., et al. (2014) ALK, ROS1 and RET Fusions in 1139 Lung Adenocarcinomas: A Comprehensive Study of Common and Fusion Pattern-Specific Clinicopathologic, Histologic and Cytologic Features. Lung Cancer, 84, 121-126. http://dx.doi.org/10.1016/j.lungcan.2014.02.007

[18] Bepler, G., Zinner, R.G., Moon, J., Calhoun, R., Kernstine, K., Williams, C.C., et al. (2014) A Phase 2 Cooperative Group Adjuvant Trial Using a Biomarker-Based Decision Algorithm in Patients with Stage I Non-Small Cell Lung Cancer (SWOG-0720, NCT00792701). Cancer, 120, 2343-2351. http://dx.doi.org/10.1002/cncr.28714

[19] Dong, X., Hao, Y., Wei, Y., Yin, Q., Du, J. and Zhao, X. (2014) Response to First-Line Chemotherapy in Patients with Non-Small Cell Lung Cancer According to RRM1 Expression. PLoS ONE, 9, e92320. http://dx.doi.org/10.1371/journal.pone.0092320

[20] Jürgens, J., Engel-Riedel, W., Prickartz, A., Ludwig, C., Schildgen, O., Tillmann, R.L., et al. (2014) Combined Point Mutation in KRAS or EGFR Genes and EML4-ALK Translocation in Lung Cancer Patients. Future Oncology, 10, 529-532. http://dx.doi.org/10.2217/fon.13.194

[21] Azijli, K., van Roosmalen, I.A., Smit, J., Pillai, S., Fukushima, M., de Jong, S., et al. (2014) The Novel Thymidylate Synthase Inhibitor Trifluorothymidine (TFT) and TRAIL Synergistically Eradicate Non-Small Cell Lung Cancer Cells. Cancer Chemotherapy and Pharmacology, 73, 1273-1283. http://dx.doi.org/10.1007/s00280-014-2465-1

[22] Murgu, S. and Colt, H. (2013) Role of the Pulmonologist in Ordering Post-Procedure Molecular Markers in Non-Small-Cell Lung Cancer: Implications for Personalized Medicine. Clinical Lung Cancer, 14, 609-626. http://dx.doi.org/10.1016/j.cllc.2013.04.002

[23] Lindeman, N.I., Cagle, P.T., Beasley, M.B., Chitale, D.A., Dacic, S., Giaccone, G., et al. (2013) Molecular Testing Guideline for Selection of Lung Cancer Patients for EGFR and ALK Tyrosine Kinase Inhibitors: Guideline from the College of American Pathologists, International Association for the Study of Lung Cancer, and Association for Molecular Pathology. Journal of Thoracic Oncology, 8, 823-859. http://dx.doi.org/10.1097/JTO.0b013e318290868f

[24] Murakami, Y., Mitsudomi, T. and Yatabe, Y. (2012) A Screening Method for the ALK Fusion Gene in NSCLC. Frontiers in Oncology, 2, 24. http://dx.doi.org/10.3389/fonc.2012.00024

[25] Institut National du Cancer (INCA). Rapport Scientifique 2010-2011.

[26] Coate, L.E. and Leighl, N.B. (2011) How Affordable Are Targeted Therapies in Non-Small Cell Lung Cancer. Current Treatment Options in Oncology, 12, 1-11. http://dx.doi.org/10.1007/s11864-010-0137-x

[27] Rapport budget année 2013 Ministère de la Santé. République Tunisienne. 3-4.

[28] Lewis, J.R., Lipworth, W.L., Kerridge, I.H. and Day, R.O. (2013) The Economic Evaluation of Personalized Oncology Medicines: Ethical Challenges. Medical Journal of Australia, 199, 471-473. 